\title{
Is Platelet Activity Important in Acute Intracerebral Hemorrhage?
}

\author{
Christine A. C. Wijman
}

Published online: 28 August 2009

(C) Humana Press Inc. 2009

Intracerebral hemorrhage (ICH) volume is a critical determinant of ICH outcome. ICH volume growth is common during the first hours following ICH onset and correlates with poor outcome [1,2]. Factors that have been associated with ICH expansion include decreased time from symptom onset to the initial brain $\mathrm{CT}$, larger ICH volume on the initial brain $\mathrm{CT}$, the presence of contrast extravasation on CT angiography, and oral anticoagulant use [3-6]. Other potentially important factors that warrant further study include increased serum glucose, increased serum creatinine, low serum cholesterol, and elevated blood pressure [7, 8].

It is biologically plausible that any factor that adversely affects hemostasis is a potential contributor to hematoma expansion and worse ICH outcome. Patients who use oral anticoagulants have larger ICH volumes on admission, more hematoma growth and worse outcomes [5]. However, studies that have evaluated the relationship between prior antiplatelet agent use and ICH outcome have yielded conflicting results [9-12]. Roquer et al. [9] reported that previous use of antiplatelet agents was an independent predictor of 30-day mortality in 194 patients with supratentorial ICH. In a population-based study of 208 patients with ICH in Finland, Saloheimo et al. [10] also found that regular aspirin use was associated with increased mortality at 3 months. In contrast, Foerch et al. [12] studied 1,691 ICH patients from a prospective registry in Germany and found that pre-hospital antiplatelet use was associated with poor outcome in univariate analysis, however, it was not an

\section{A. C. Wijman $(\square)$}

Department of Neurology and Neurological Sciences,

Stanford University Medical Center, Stanford Stroke Center,

701 Welch Road, B325, Palo Alto, CA 94304, USA

e-mail: cwijman@stanford.edu independent predictor of ICH outcome after adjustment for age and pre-hospital status. These results raise the question whether prior antiplatelet use worsens $\mathrm{ICH}$, or whether it identifies a subpopulation of ICH patients that tends to have more baseline co-morbidities and therefore is more likely to fare worse following ICH.

Data from three well-designed prospective clinical studies suggest that if there is a biological association between prior antiplatelet use and ICH volume growth, it is modest at best. Brott et al. [1] imaged 103 ICH patients within $3 \mathrm{~h}$ of symptom onset and again $20 \mathrm{~h}$ later and did not find an association between prior antiplatelet use and ICH volume growth. In 282 prospectively enrolled patients in the placebo arm of the cerebral hemorrhage and NXY059 treatment (CHANT) trial who were imaged within $6 \mathrm{~h}$ of symptom onset, use of antiplatelet agents was not associated with increased hematoma volumes at presentation, nor with hemorrhage expansion or worse outcome at 90 days [11]. In contrast, patients in the same CHANT trial who were on oral anticoagulants did exhibit larger baseline hematoma volumes, more ICH expansion and worse outcomes [5]. In an exploratory analysis of the phase II trial of recombinant activated factor VII (rFVIIa) in patients with $\mathrm{ICH}$, which included five models of change in hemorrhage volume, prior use of antiplatelet agents was associated with ICH expansion in only one of the five models, whereas treatment with rFVIIa and a longer time from onset to baseline $\mathrm{CT}$ were related to a decrease in ICH expansion in all models [7]. Thus, the current available data do not support an important effect of prior antiplatelet use on hematoma growth.

With this as a background, the research published this year by Naidech et al. [13-15] on the occurrence of reduced platelet activity in ICH patients is of interest. The investigators found in a relatively small prospective cohort 
of patients with primary ICH that volume expansion, intraventricular hemorrhage, and 3 month mortality were more common in patients with reduced platelet activity on presentation measured by a platelet function assay [13, 14]. They also found that platelet activity improved after platelet transfusion [13]. These findings from a single center deserve further study.

In their article published in Neurocritical Care, Naidech et al. [15] reported the presence of reduced platelet function in $33(46 \%)$ and $27(38 \%)$ of $72 \mathrm{ICH}$ patients measured by two different platelet function assays. Of these, 42 and $52 \%$ of patients, respectively, were not known to take antiplatelet agents. This finding raises the question whether some patients take antiplatelet agents at the onset of headache as hypothesized by the authors, or whether acute ICH itself reduces platelet activity. Studying a control group, for example patients with traumatic ICH, and performing serial platelet function tests in spontaneous ICH patients might provide further insights. It is of concern that Naidech et al. found poor agreement between the two platelet function assays that they used (kappa $=0.26$, $P=0.07)$ and there was also no agreement between reported antiplatelet use and decreased platelet function for one of the assays. These findings hamper interpretation of their results and call into question the accuracy of these tests for assessing platelet function in ICH patients.

Although the findings of Naidech et al. are intriguing, it is not yet prime-time for testing a therapeutic intervention aimed at improving platelet activity based on the current available data. We will first need to be convinced that any one of the currently available platelet function assays indeed identifies patients who are at risk for ICH expansion and poor outcome. Only then can we further explore the question whether interventions aimed at improving platelet activity will favorably affect ICH outcome.

\section{References}

1. Brott T, Broderick J, Kothari R, Barsan W, Tomsick T, Sauerbeck L, et al. Early hemorrhage growth in patients with intracerebral hemorrhage. Stroke. 1997;28:1-5.
2. Davis SM, Broderick J, Hennerici M, Brun NC, Diringer MN, Mayer SA, et al. Hematoma growth is a determinant of mortality and poor outcome after intracerebral hemorrhage. Neurology. 2006;66:1175-81.

3. Wada R, Aviv RI, Fox AJ, Sahlas DJ, Gladstone DJ, Tomlinson $\mathrm{G}$, et al. CT angiography "spot sign" predicts hematoma expansion in acute intracerebral hemorrhage. Stroke. 2007;38: 1257-62.

4. Goldstein JN, Fazen LE, Snider R, Schwab K, Greenberg SM, Smith EE, et al. Contrast extravasation on $\mathrm{CT}$ angiography predicts hematoma expansion in intracerebral hemorrhage. Neurology. 2007;68:889-94.

5. Cucchiara B, Messe S, Sansing L, Kasner S, Lyden P, CHANT Investigators. Hematoma growth in oral anticoagulant related intracerebral hemorrhage. Stroke. 2008;39:2993-6.

6. Fujii Y, Takeuchi S, Sasaki O, Minakawa T, Tanaka R. Multivariate analysis of predictors of hematoma enlargement in spontaneous intracerebral hemorrhage. Stroke. 1998;29:1160-6.

7. Broderick JP, Diringer MN, Hill MD, Brun NC, Mayer SA, Steiner $\mathrm{T}$, et al. Determinants of intracerebral hemorrhage growth: an exploratory analysis. Stroke. 2007;38:1072-5.

8. Anderson CS, Huang Y, Wang JG, Arima H, Neal B, Peng B, et al. Intensive blood pressure reduction in acute cerebral haemorrhage trial (INTERACT): a randomised pilot trial. Lancet Neurol. 2008;7:391-9.

9. Roquer J, Rodríguez Campello A, Gomis M, Ois A, Puente V, Munteis E. Previous antiplatelet therapy is an independent predictor of 30-day mortality after spontaneous supratentorial intracerebral hemorrhage. J Neurol. 2005;252:412-6.

10. Saloheimo P, Ahonen M, Juvela S, Pyhtinen J, Savolainen ER, Hillbom M. Regular aspirin-use preceding the onset of primary intracerebral hemorrhage is an independent predictor for death. Stroke. 2006;37:129-33.

11. Sansing LH, Messe SR, Cucchiara BL, Cohen SN, Lyden PD, Kasner SE, et al. Prior antiplatelet use does not affect hemorrhage growth or outcome after ICH. Neurology. 2009;72:1397-402.

12. Foerch C, Sitzer M, Steinmetz H, Neumann-Haefelin T. Pretreatment with antiplatelet agents is not independently associated with unfavorable outcome in intracerebral hemorrhage. Stroke. 2006;37:2165-7.

13. Naidech AM, Jovanovic B, Liebling S, Garg RK, Bassin SL, Bendok BR, et al. Reduced platelet activity is associated with early clot growth and worse 3-month outcome after intracerebral hemorrhage. Stroke. 2009;40:2398-401.

14. Naidech AM, Bernstein RA, Levasseur K, Bassin SL, Bendok $\mathrm{BR}, \mathrm{Batjer} \mathrm{HH}$, et al. Platelet activity and outcome after intracerebral hemorrhage. Ann Neurol. 2009;65:352-6.

15. Naidech AM, Bassin SL, Bernstein RA, Batjer HH, Alberts MJ, Lindholm PF, Bleck TP. Reduced platelet activity is more common than reported anti-platelet medication use in patients with intracerebral hemorrhage. Neurocrit Care. 2009. [Epub ahead of print]. 